This item is the archived peer-reviewed author-version of:

\title{
Fertile forests produce biomass more efficiently
}

\section{Reference:}

Vicca Sara, Luyssaert Sebastiaan, Peñuelas J., Campioli Matteo, Janssens Ivan, et al..- Fertile forests produce biomass more efficiently

Ecology letters - ISSN 1461-0248 - 15:6(2012), p. 520-526

Full text (Publishers DOI): http://dx.doi.org/doi:10.1111/j.1461-0248.2012.01775.x

Handle: http://hdl.handle.net/10067/969720151162165141 
49

\section{Fertile forests produce biomass more efficiently}

Vicca $\mathrm{S}^{1}$, Luyssaert $\mathrm{S}^{2}$, Peñuelas $\mathrm{J}^{3}$, Campioli $\mathrm{M}^{1}$, Chapin FS $\mathrm{III}^{4}$, Ciais $\mathrm{P}^{2}$, Heinemeyer $\mathrm{A}^{5}$, Högberg $\mathrm{P}^{6}$, Kutsch $\mathrm{WL}^{7}$, Law BE ${ }^{8}$, Malhi $\mathrm{Y}^{9}$, Papale $\mathrm{D}^{10}$, Piao SL ${ }^{11}$, Reichstein $\mathrm{M}^{12}$, Schulze $\mathrm{ED}^{12}$, Janssens IA ${ }^{1}$.

1 Department of Biology, University of Antwerp, 2610 Wilrijk, Belgium

2 LSCE CEA-CNRS-UVSQ, Orme des Merisiers, F-91191 Gif-sur-Yvette, France

3 Global Ecology Unit CREAF-CEAB-CSIC, CREAF (Center for Ecological Research and Forestry Applications), Edifici C, Universitat Autònoma Barcelona, 08193 Bellaterra, Spain

4 Institute of Arctic Biology, University of Alaska Fairbanks, Fairbanks, AK 99775, USA

5 Environment Department, Centre for Terrestrial Carbon Dynamics (York-Centre) at the Stockholm Environment Institute, University of York, YO10 5DD, York, UK

6 Department of Forest Ecology and Management, Swedish University of Agricultural Sciences (SLU), SE-901 83 Umeå, Sweden.

7 Johann Heinrich von Thünen Institut, Institute of Agricultural Climate Research, 38116 Braunschweig, Germany

8 Forest Ecosystems and Society Department, Oregon State University, Corvallis, Oregon 97331, USA

9 Environmental Change Institute, School of Geography and the Environment, University of Oxford, Oxford OX1 3QY, UK

10 Department of Forest Environment and Resources, University of Tuscia, I-01100 Viterbo, Italy

11 College of Urban and Environmental Sciences and KLESPME, Peking University, Beijing 100871, China

12 Max Planck Institute for Biogeochemistry, PO Box 100164, D-07701 Jena, Germany

Article type: Ideas and Perspectives

Short running title: Forests' biomass production efficiency

Email: SV sara.vicca@ua.ac.be; SL sebastiaan.luyssaert@1sce.ipsl.fr; JP

josep.penuelas@uab.cat; MC matteo.campioli@ua.ac.be; FSC terry.chapin@alaska.edu; PC philippe.ciais@cea.fr; AH andreas.heinemeyer@york.ac.uk; PH peter.hogberg@slu.se; WK werner.kutsch@ vti.bund.de; BEL bev.law@oregonstate.edu; YM yadvinder.malhi@ouce.ox.ac.uk; DP darpap@unitus.it; SLP slpiao@pku.edu.cn; MR markus.reichstein@bgc-jena.mpg.de; EDS dschulze@ bgc-jena.mpg.de; IAJ ivan.janssens@ua.ac.be

Authorship: SV, SL, JP and IAJ conceived the paper; SV performed the analyses and wrote the paper; all authors contributed substantially to discussions and revisions.

Corresponding author: Sara Vicca, universiteitsplein 1, 2610 Wilrijk, Belgium; sara.vicca@ua.ac.be; tel.: +3232652282; fax: +3232652271

Keywords: global forest database; biomass production; photosynthesis; biomass production efficiency; carbon allocation; nutrients; root symbionts

Word count: abstract: 150; main text: 2259; whole manuscript: 5936

Figures: 3 (of which one in BOX 1)

Tables: 3

Boxes: 2

Number of references: 39 


\section{Abstract}

51 Trees with sufficient nutrition are known to allocate carbon preferentially to aboveground plant 52 parts. Our global study of 49 forests revealed an even more fundamental carbon allocation 53 response to nutrient availability: forests with high nutrient availability use $58 \pm 3 \%$ (mean \pm SE; 17 54 forests) of their photosynthates for plant biomass production, while forests with low nutrient 55 availability only convert $42 \pm 2 \%$ (mean \pm SE; 19 forests) of annual photosynthates to biomass. 56 This nutrient effect largely overshadows previously observed differences in carbon allocation 57 patterns among climate zones, forest types and age classes. If forests with low nutrient 58 availability use $16 \pm 4 \%$ less of their photosynthates for plant growth, what are these used for? 59 Current knowledge suggests that lower biomass production per unit photosynthesis in forests 60 with low- versus forests with high nutrient availability reflects not merely an increase in plant 61 respiration, but likely results from reduced carbon allocation to unaccounted components of net 62 primary production, particularly root symbionts. 
66 Plant physiologists often argue that, across species and biomes, plants respire a nearly constant

67 fraction - approximately 50\% - of the carbon taken up during photosynthesis (GPP) (Gifford

68 1994, 1995; Dewar et al. 1998; Waring et al. 1998; Enquist et al. 2007; Van Oijen et al. 2010).

69 The remaining carbon - termed net primary production (NPP) - is converted into plant biomass

70 and other complex molecules used for multiple purposes (e.g., root exudation, production of

71 volatile organic compounds). The relative constancy of the partitioning of GPP into autotrophic

72 respiration $(\mathrm{Ra})$ and NPP would reflect the interdependence of respiration and photosynthesis.

73 Respiration depends on the substrate provided by photosynthesis, which in turn relies on

74 respiration to provide the energy required for construction of complex compounds such as carbon

75 skeletons for protein synthesis (Krömer 1995; Hoefnagel et al. 1998). Unfortunately, the

76 verification of this theory at the ecosystem scale is severely hampered by the fact that NPP and

$77 \mathrm{Ra}$ are difficult to quantify for the entire ecosystem. While Ra occurs in every living plant cell

78 within the ecosystem, NPP includes numerous carbon-consuming processes (plant growth, root

79 exudation, carbon allocation to symbionts and production of volatile organic compounds). The

80 paucity of accurate data on forest ecosystem Ra and NPP globally explains why the partitioning

81 of GPP at the ecosystem scale remains poorly understood.

82

83 Because biomass production constitutes the largest fraction of NPP, biomass production is 84 commonly used as a proxy for NPP (Waring et al. 1998; DeLucia et al. 2007; Drake et al. 2011;

85 Goulden et al. 2011). In contrast to theoretical argumentations for a constrained NPP-to-GPP 86 ratio (Dewar et al. 1998; Van Oijen et al. 2010), field measurements in forests revealed 87 substantial variation in the biomass production-to-GPP ratio (DeLucia et al. 2007). The biomass 88 production-to-GPP ratio was reported to be higher in forests of the temperate zone, in particular 
89 in broadleaved temperate forests (DeLucia et al. 2007), and to decrease with increasing stand age

90 (DeLucia et al. 2007; Goulden et al. 2011). These results are, however, tentative because the

91 effect of stand age is confounded with forest type and climate zone; the majority of the young

92 forests is located in the temperate zone (DeLucia et al. 2007; Drake et al. 2011).

94 Because biomass production and GPP data have become increasingly available in recent years,

95 we revisited the global variation in ecosystem-scale carbon partitioning patterns using a global 96 forest database (Luyssaert et al. 2007). For the current study, we selected only those forests that 97 provided estimates of above- and belowground biomass production and GPP that were 98 independent from each other (i.e., biomass production estimates via biometry and GPP via eddy 99 covariance or in a few cases using a model). Whenever necessary, biomass production estimates 100 of the resulting 49 forests were complemented with estimates of missing biomass components. 101 This procedure did not affect our conclusions (see Appendix S1). Further detailed information 102 regarding the dataset is provided in Appendix S1.

103

104 Last, to make a clear distinction with the NPP-to-GPP ratio (which comprises not only plant 105 biomass production, but also production of volatile organic compounds, root exudates and root 106 symbionts), we here introduce the term Biomass Production Efficiency (BPE) when referring to 107 the biomass production-to-GPP ratio. Biomass production contains all the biomass produced 108 within a year irrespective of whether this biomass dies within the same or subsequent years.

110 Variables explaining variation in biomass production efficiency

111 Previous studies focussed on climate, forest type and stand age to explain the observed 112 differences in the biomass production-to GPP ratio among forests (DeLucia et al. 2007; Goulden 
113 et al. 2011). Nutrient availability and forest management (unmanaged versus management 114 involving harvesting, thinning, etc.) significantly affect allocation patterns in forests (Shan et al. 115 2001; Litton et al. 2007; LeBauer \& Treseder 2008). Plants exposed to ample nutrients invest 116 relatively less carbon in roots, while plants growing under low nutrient availability use relatively 117 more carbon for root growth at the expense of aboveground growth (Chapin 1980). Forest 118 management also has been found to decrease root-to-shoot ratios (Shan et al. 2001) and both 119 nutrient availability and forest management are thus potentially important factors influencing 120 BPE. So far, however, they have not been assessed.

121

122 While information on climate, forest type, stand age and management practices is easily 123 available, measured nutrient availability is not. Estimation of comparable nutrient availability is 124 not a simple task and requires standardized measurements. Effective plant nutrient availability 125 depends on multiple factors besides soil nutrient content (soil texture, $\mathrm{pH}$, cation exchange 126 capacity, moisture), such that it can differ substantially among sites with, for example, similar 127 soil nitrogen contents but different soil texture. Furthermore, comparison of nutrient availability 128 among ecosystems requires consideration of all plant nutrients, and not only nitrogen, as was 129 demonstrated for a range of hardwood forests in northern United States and Canada 130 (Vadeboncoeur 2010). Unfortunately, such a uniform estimation of nutrient availability in forests 131 across the globe does not currently exist. In order to test whether BPE increased with increasing 132 nutrient availability, we therefore assigned each of the 49 forests in our dataset to one of three 133 categories: low-, medium-, or high-nutrient availability following the information available in 134 literature (see Table S3 in Appedix S2). 
Although this classification is not a simple task, information for forests of the low and high

137 nutrient availability class was generally very clear. Forests of the low nutrient availability class were typically located on soils with extremely low nutrient content due to weathering, leaching,

139 or low mineralization rates. In contrast, some of the forests assigned to the high nutrient 140 availability class grew on former (fertilized) agricultural land, while others were located on soil

141 types that are renowned as very fertile (see Appendix S2). Moreover, for 14 of the 17 forests of 142 the high nutrient availability class, an explicit statement of the nutrient status was provided in 143 publications (see Appendix S2). Last, a sensitivity analysis revealed that potential 144 misclassification of sites with relatively little information would not influence our conclusions 145 (Table S4).

As expected, differences in absolute biomass production between forests of similar nutrient status growing in different climate zones were large (Tables 1 and 3), but these differences were entirely attributable to differences in GPP, with BPE changing little within nutrient classes (Table 1). In contrast, the large differences in biomass production among temperate-zone forests of 151 different nutrient availability were not solely due to variation in GPP. Whereas GPP did not 152 significantly differ between temperate forests of the low and high nutrient availability class $153(+31 \%, \mathrm{p}=0.19$; Table 1$)$, biomass production was $78 \%$ higher in temperate forests of the high 154 nutrient availability class than in temperate forests with low nutrient availability $(\mathrm{p}=0.01$; Table 155 1). This disproportionate increase in biomass production relative to GPP was most pronounced in 156 woody biomass, with three-fold higher aboveground wood production at high compared to low 157 nutrient availability $(\mathrm{p}=0.02$; Table 2$)$, while foliage and root biomass production remained 158 largely unchanged (Table $2 ; \mathrm{p}=0.49$ and $\mathrm{p}=0.83$, respectively). As a consequence of the much 159 larger nutrient effect on biomass production compared to GPP, BPE was $35 \pm 9 \%$ (mean $\pm \mathrm{SE}$ ) 
163 In order to test whether nutrient availability was indeed the key factor explaining variation in 164 BPE, we performed a stepwise regression analysis including climate zone (boreal, temperate and 165 tropical), forest type (coniferous, broadleaved and mixed), stand age, nutrient availability and 166 forest management (i.e., unmanaged or managed). For more information regarding stepwise 167 regression analysis, see Cohen (1991), Derr \& Everitt (2002) and Appendix S4. In contrast to 168 results reported in other analyses (DeLucia et al. 2007; Goulden et al. 2011), analysis of our data 169 set indicated that neither climate zone, nor forest type or stand age significantly affected BPE, 170 whereas nutrient availability affected BPE highly significantly $(\mathrm{p}<0.01)$. Independently of 171 climate or forest type, forests with high nutrient availability allocated on average $58 \pm 3 \%$

172 (mean \pm SE of 17 forests) of their photosynthates to biomass production, whereas forests with low

173 nutrient availability used on average only $42 \pm 2 \%$ (mean \pm SE of 19 forests) of their photosynthates 174 for biomass production (i.e., slopes of lines in Fig. $1 ; \mathrm{p}<0.01$ for low versus high nutrient 175 availability). This result of nutrient availability being the primary determinant of BPE was 176 confirmed also by other statistical tests (see Appendix S4), irrespective of whether or not we 177 accounted for measurement uncertainties.

179 Figure 2A further suggests that previously reported differences in BPE among boreal, temperate 180 and tropical forests (DeLucia et al. 2007) were introduced by the uneven distribution of forests 181 with high nutrient availability (with higher BPE) across the globe, being heavily biased towards 182 the temperate zone. Likewise, the previously reported difference between coniferous and 183 broadleaved forests (DeLucia et al. 2007) was not apparent when taking nutrient availability into 
account, probably because nutrient-rich soils were occupied more by broadleaved than by 185 coniferous forests (Fig. 2C).

187 In addition to nutrient availability, which was by far the dominant determinant of variation in 188 BPE, management also affected BPE significantly according to the stepwise regression analysis $189(\mathrm{p}=0.02)$. Managed forests exhibited higher BPE than unmanaged forests for both low and high 190 nutrient availability classes (Fig. 2B), but quantification of this effect remains premature because 191 of the uneven distribution of unmanaged and managed forests among nutrient classes (Fig. 2B). 192 Nonetheless, the distinction between unmanaged and managed forests appeared an important 193 factor in the relationship between stand age and BPE, because the tendency for a negative age 194 effect on BPE as observed in Figure 2D only became significant $(\mathrm{p}=0.04)$ if management regime 195 was not included in the stepwise regression analysis.

\section{Underlying mechanisms}

198 The significantly higher BPE in forests with high nutrient availability as compared to forests of 199 the medium or low nutrient availability class implies that either a smaller fraction of GPP is being 200 respired in the forests with high nutrient availability, or a smaller fraction of GPP is partitioned to 201 unaccounted NPP components (VOC emissions, root symbionts, root exudation), or a 202 combination of both. Because estimates of carbon transfers to volatile organic compounds 203 (VOC), and to root exudates and symbionts are not available for any of the forests in our 204 database, the only way to test why BPE differs between forests of different nutrient availability is 205 by comparing estimates of the Ra-to-GPP ratio. Many pitfalls arise when measuring Ra at the 206 ecosystem level (Ryan et al. 1997; Amthor \& Baldocchi 2001), ecosystem Ra is therefore most 
207 often estimated as the residual of GPP minus biomass production, rendering these Ra estimates 208 useless to test whether or not variation in the ratio of Ra to GPP could explain variation in BPE.

210 Only 11 of our sites provided ecosystem Ra and GPP estimates that were independent from 211 biomass production. These 11 forests revealed no effect of nutrient availability on the Ra-to-GPP 212 ratio (see Fig. 3 in BOX 1), suggesting that variation in the ratio of Ra to GPP does not explain 213 the higher BPE in nutrient-rich forests. Obviously, the limited data availability constrains the 214 robustness of this analysis. Nonetheless, in addition to this lack of empirical evidence for a 215 difference in the Ra-to-GPP ratio among nutrient availability classes, it appears unlikely that 216 nutrient-rich forests that grow faster (which would lead to more growth respiration) and that 217 likely exhibit higher protein levels (which would lead to higher maintenance respiration would 218 exhibit considerably lower Ra-to-GPP ratios than nutrient-poor forests (see BOX 1 for a more 219 thorough elaboration of the underlying rationale). We therefore hypothesize that the unmeasured 220 NPP components explain the difference in BPE among nutrient availability classes. Particularly 221 root symbionts are a plausible candidate (BOX 2).

223 Finally, managed forests exhibited higher BPE than unmanaged forests for both the high and low 224 nutrient availability class. Management via thinning sometimes implies removal of biomass 225 expected to grow sub-optimally, such as suppressed trees with large autotrophic respiration 226 relative to GPP. Such removals may decrease the relative amount of maintenance respiration and 227 consequently also the Ra-to-GPP ratio. The higher BPE in managed than in unmanaged forests 228 may thus reflect this reduced Ra-to-GPP ratio. In addition, managed forests may exhibit higher 229 BPE than unmanaged forests because frequent anthropogenic disturbances tend to (further) increase nutrient availability. 
232 Our analysis of 49 forest sites where biomass production and GPP were independently measured 233 revealed that nutrient availability may be the unifying mechanism controlling the ratio of biomass 234 production-to-GPP that encompasses climate, forest type, and stand age as influencing factors. 235 The carbon sink potential of forests largely depends on how carbon taken up during 236 photosynthesis is partitioned. Photosynthates partitioned to $\mathrm{Ra}$ do not contribute to carbon 237 sequestration but those converted into long-lived biomass do contribute. The observed pattern of 238 higher carbon partitioning to plant biomass with increasing nutrient availability thus adds to our 239 understanding of the processes governing long-term carbon sequestration in forests and may have 240 far-reaching consequences for carbon cycle management. Further research is needed to verify 241 how the higher BPE in forests with high nutrient availability, together with the previously 242 reported decrease of soil organic matter decomposition in response to fertilization (Janssens et al. 243 2010) determines ecosystem carbon sequestration.

245 It remains unresolved whether the increased partitioning to biomass production relative to GPP 246 associated with higher nutrient availability is related to a lower Ra-to-GPP ratio or to a small 247 fraction of NPP going to typically unaccounted for components such as VOC production, root 248 symbionts, and root exudates in forests of high versus low nutrient availability. The present study 249 points in the direction of the latter (see also BOX 2). Future (large-scale) experiments in which 250 nutrient availability is manipulated and where all measurements needed to unravel carbon 251 partitioning are made (i.e., independent estimates for all NPP components, GPP and Ra) would 252 help resolve these questions. 


\section{Acknowledgements}

256 We thank all site investigators, their funding agencies, the various regional flux networks 257 (Afriflux, AmeriFlux, AsiaFlux, CarboAfrica, CarboEurope-IP, ChinaFlux, Fluxnet-Canada, 258 KoFlux, LBA, NECC, OzFlux, TCOS-Siberia, USCCC), development of measurement and data 259 submission protocols (funded by Office of Science (BER), U.S. Dept of Energy), and the Fluxnet 260 project, whose work and support is essential for obtaining the measurements without which the 261 type of integrated analyses conducted in this study would not be possible. The authors 262 acknowledge Arie Weeren for statistical help and the FP7 GHG Europe project for financial 263 support. SV is a post-doctoral research associate of the Fund for Scientific Research - Flanders, 264 SL is funded by ERC Starting Grant 242564 and JP by grants Consolider Ingenio Montes 265 CSD2008-00040 and CGL2010-17172.

\section{SUPPORTING INFORMATION}

268 Additional Supporting Information may be found in the online version of this article:

270 Appendix S1: Detailed information about the data used

271 Appendix S2: Nutrient classification

272 Appendix S3: Additional discussion regarding uncertainties on fine root production estimates

273 Appendix S4: Detailed information about statistical analyses

275 As a service to our authors and readers, this journal provides supporting information supplied by 276 the authors. Such materials are peer-reviewed and may be re-organized for online delivery, but 277 are not copy-edited or typeset. Technical support issues arising from supporting information 278 (other than missing files) should be addressed to the authors. 


\section{References}

282 Amthor, J.S. (2000). The McCree-de Wit-Penning de Vries-Thornley respiration paradigms: 30 283 years later. Ann. Bot., 86, 1-20.

284 Amthor, J.S. \& Baldocchi, D.D. (2001). Terrestrial higher plant respiration and net primary 285 production. In: Terrestrial Global Productivity (eds. Roy J, Saugier B \& Mooney HA). Academic 286 Press San Diego, pp. 33-59.

287 Atkin, O.K., Scheurwater, I. \& Pons, T.L. (2007). Respiration as a percentage of daily 288 photosynthesis in whole plants is homeostatic at moderate, but not high, growth temperatures. 289 New Phytol., 174, 367-380.

290 Burton, A.J., Pregitzer, K.S., Ruess, R.W., Hendrik, R.L. \& Allen, M.F. (2002). Root respiration 291 in North American forests: effects of nitrogen concentration and temperature across biomes. 292 Oecologia, 131, 559-568.

293 Campbell, C., Atkinson, L., Zaragoza-Castells, J., Lundmark, M., Atkin, O. \& Hurry, V. (2007). 294 Acclimation of photosynthesis and respiration is asynchronous in response to changes in 295 temperature regardless of plant functional group. New Phytol., 176, 375-389.

296 Chapin, F.S. (1980). The mineral nutrition of wild plants. Annu. Rev. Ecol. Syst., 11, 233-260.

297 Courty, P.E., Buée, M., Diedhiou, A.G., Frey-Klett, P., Le Tacon, F., Rineau, F., et al. (2010). 298 The role of ectomycorrhizal communities in forest ecosystem processes: New perspectives and 299 emerging concepts. Soil Biol. Biochem., 42, 679-698.

300 DeLucia, E.H., Drake, J.E., Thomas, R.B. \& Gonzalez-Meler, M. (2007). Forest carbon use 301 efficiency: is respiration a constant fraction of gross primary production? Global Change Biol., 302 $13,1157-1167$. 

304 carbon use efficiencies. Plant Cell Environ., 21, 573-588.

305 Drake, J.E., Davis, S.C., Raetz, L.M. \& DeLucia, E.H. (2011). Mechanisms of age-related 306 changes in forest production: the influence of physiological and successional changes. Global 307 Change Biol., 17, 1522-1535.

308 Enquist, B.J., Kerkhoff, A.J., Huxman, T.E. \& Economo, E.P. (2007). Adaptive differences in 309 plant physiology and ecosystem paradoxes: insights from metabolic scaling theory. Global 310 Change Biol., 13, 591-609.

311 Gifford, R.M. (1994). The global carbon cycle: a viewpoint on the missing sink. Austr. J. Plant 312 Physiol., 21, 1-15.

313 Gifford, R.M. (1995). Whole plant respiration and photosynthesis of wheat under increased $\mathrm{CO}_{2}$ 314 concentration and temperature: long-term vs. short-term distinctions for modelling. Global 315 Change Biol., 1, 385-396.

316 Goulden, M.L., McMillan, A.M.S., Winston, G.C., Rocha, A.V., Manies, K.L., Harden, J.W., et 317 al. (2011). Patterns of NPP, GPP, respiration, and NEP during boreal forest succession. Global 318 Change Biol., 17, 855-871.

319 Heinemeyer, A., Wilkinson, M., Vargas, R., Subke, J.A., Casella, E., Morison, J.I.L., et al. 320 (2012). Exploring the "overflow tap" theory: linking forest soil $\mathrm{CO}_{2}$ fluxes and individual 321 mycorrhizosphere components to photosynthesis. Biogeosci., 9, 79-95.

322 Hobbie, E.A. (2006). Carbon allocation to ectomycorrhizal fungi correlates with belowground 323 allocation in culture studies. Ecology, 87, 563-569.

324 Hoefnagel, M.H.N., Atkin, O.K. \& Wiskich, J.T. (1998). Interdependence between chloroplasts 325 and mitochondria in the light and the dark. Biochim. Biophys. Acta, 1366, 235-255. 
Högberg, M.N., Baath, E., Nordgren, A., Arnebrant, K. \& Högberg, P. (2003). Contrasting 327 effects of nitrogen availability on plant carbon supply to mycorrhizal fungi and saprotrophs - a 328 hypothesis based on field observations in boreal forest. New Phytol., 160, 225-238.

329 Högberg, M.N., Briones, M.J.I., Keel, S.G., Metcalfe, D.B., Campbell, C., Midwood, A.J., et al. 330 (2010). Quantification of effects of season and nitrogen supply on tree below-ground carbon 331 transfer to ectomycorrhizal fungi and other soil organisms in a boreal pine forest. New Phytol., $332187,485-493$.

333 Janssens, I.A., Dieleman, W., Luyssaert, S., Subke, J.A., Reichstein, M., Ceulemans, R., et al. 334 (2010). Reduction of forest soil respiration in response to nitrogen deposition. Nature Geosci., 3 , $335 \quad 315-322$.

336 Krömer, S. (1995). Respiration during photosynthesis. Annu. Rev. Plant Physiol. Plant Mol. 337 Biol., 46, 45-70.

338 LeBauer, D.S. \& Treseder, K.K. (2008). Nitrogen limitation of net primary productivity in 339 terrestrial ecosystems is globally distributed. Ecology, 89, 371-379.

340 Lilleskov, E.A., Fahey, T.J., Horton, T.R. \& Lovett, G.M. (2002). Belowground ectomycorrhizal 341 fungal community change over a nitrogen deposition gradient in Alaska. Ecology, 83, 104-115.

342 Litton, C.M., Raich, J.W. \& Ryan, M.G. (2007). Carbon allocation in forest ecosystems. Global 343 Change Biol., 13, 2089-2109.

344 Loveys, B.R., Atkinson, L.J., Sherlock, D.J., Roberts, R.L., Fitter, A.H. \& Atkin, O.K. (2003). 345 Thermal acclimation of leaf and root respiration: an investigation comparing inherently fast- and 346 slow-growing plant species. Global Change Biol., 9, 895-910.

347 Luyssaert, S., Inglima, I., Jung, M., Richardson, A.D., Reichsteins, M., Papale, D., et al. (2007). $348 \mathrm{CO}_{2}$ balance of boreal, temperate, and tropical forests derived from a global database. Global 349 Change Biol., 13, 2509-2537. 
351 carbon cost: a global-scale analysis of autotrophic respiration. Ecology, 91, 652-661.

352 Read, D.J. \& Perez-Moreno, J. (2003). Mycorrhizas and nutrient cycling in ecosystems - a 353 journey towards relevance? New Phytol., 157, 475-492.

354 Reich, P.B., Walters, M.B., Ellsworth, D.S., Vose, J.M., Volin, J.C., Gresham, C., et al. (1998).

355 Relationships of leaf dark respiration to leaf nitrogen, specific leaf area and leaf life-span: a test 356 across biomes and functional groups. Oecologia, 114, 471-482.

357 Ryan, M.G., Lavigne, M.B. \& Gower, S.T. (1997). Annual carbon cost of autotrophic respiration 358 in boreal forest ecosystems in relation to species and climate. J. Geophys. Res., 102, 2887135928883.

360 Schulze, E.D. (2000). The carbon and nitrogen cycle in forest ecosystems. In: Carbon and 361 Nitrogen Cycling in European Forest Ecosystems (ed. Schulze ED). Springer, Berlin, p. 500.

362 Shan, J.P., Morris, L.A. \& Hendrick, R.L. (2001). The effects of management on soil and plant 363 carbon sequestration in slash pine plantations. J. Appl. Ecol., 38, 932-941.

364 Treseder, K.K. (2004). A meta-analysis of mycorrhizal responses to nitrogen, phosphorus, and 365 atmospheric $\mathrm{CO}_{2}$ in field studies. New Phytol., 164, 347-355.

366 Turnbull, M.H., Tissue, D.T., Griffin, K.L., Richardson, S.J., Peltzer, D.A. \& Whitehead, D. 367 (2005). Respiration characteristics in temperate rainforest tree species differ along a long-term 368 soil-development chronosequence. Oecologia, 143, 271-279.

369 Vadeboncoeur, M.A. (2010). Meta-analysis of fertilization experiments indicates multiple 370 limiting nutrients in northeastern deciduous forests. Can. J. For. Res., 40, 1766-1780.

371 van der Heijden, M.G.A., Bardgett, R.D. \& van Straalen, N.M. (2008). The unseen majority: soil 372 microbes as drivers of plant diversity and productivity in terrestrial ecosystems. Ecol. Lett., 11, $373 \quad 296-310$. 
374 Van Oijen, M., Schapendonk, A. \& Hoglind, M. (2010). On the relative magnitudes of 375 photosynthesis, respiration, growth and carbon storage in vegetation. Ann. Bot., 105, 793-797.

376 Wallenda, T. \& Kottke, I. (1998). Nitrogen deposition and ectomycorrhizas. New Phytol., 139, $377 \quad 169-187$.

378 Waring, R.H., Landsberg, J.J. \& Williams, M. (1998). Net primary production of forests: a 379 constant fraction of gross primary production? Tree Physiol., 18, 129-134.

380 


\section{BOX 1: Autotrophic respiration-to-GPP ratio}

383 The biomass production-to-photosynthesis ratio is typically used as a proxy for the ratio of net 384 primary production (NPP) to photosynthesis (GPP), termed carbon use efficiency (CUE) (e.g. 385 DeLucia et al. 2007). Alternatively, CUE can be determined using autotrophic respiration (Ra) 386 instead of biomass production, i.e., $\mathrm{CUE}=1-(\mathrm{Ra}$ GPP). Estimates of Ra (not derived from 387 biomass production measurements) are usually obtained by upscaling respiration measured on 388 parts of the vegetation (foliage, stem, branch) or by subtracting heterotrophic respiration from 389 eddy covariance-based estimates of ecosystem respiration (see e.g. Piao et al. (2010)). Estimates 390 of Ra (independent of biomass production) are less abundant than biomass production estimates, 391 which is the primary reason why we focus on the biomass production-to-GPP ratio and not on the 392 ratio of Ra to GPP. Nonetheless, we show the results of the 11 forests in our dataset that provided 393 estimates of Ra that were independent of GPP and biomass production (Fig. 3). The Ra-to-GPP 394 ratio did not significantly differ among nutrient availability classes $(\mathrm{p}=0.34$ for ANOVA with 395 nutrient availability as fixed factor), but with only two nutrient-rich forests, it is premature to 396 draw meaningful conclusions.

398 Literature on respiration measurements at organ level (root, woody tissue, foliage) also provides 399 no definite answer because both increases and decreases in the Ra-to-GPP ratio with increasing 400 nutrient availability appear possible. Autotrophic respiration ( $\mathrm{Ra})$ is typically positively related to 401 tissue nitrogen concentrations (Chapin 1980). Because photosynthesis also increases with 402 increasing nitrogen concentration, the Ra-to-GPP ratio of leaves appears relatively constant 403 across species, climates and ecosystem types (Reich et al. 1998; Loveys et al. 2003; Turnbull et 404 al. 2005; Atkin et al. 2007; Campbell et al. 2007), although under extreme conditions this ratio 
may increase (Atkin et al. 2007; Campbell et al. 2007) and potentially indicates an increase of

406 'wastage' respiration needed to discard excess energy and prevent cell damage (Amthor 2000). In

407 one study, the leaf respiration-to-photosynthesis ratio was higher in two forests suffering severe

408 nutrient limitations as compared to neighbouring less nutrient-stressed forests (Turnbull et al. 409 2005).

410

411 On the other hand, in forests with high nutrient availability, a larger fraction of photosynthates 412 typically is invested in wood compared to the fraction invested in wood in forests with low 413 nutrient availability (Litton et al. 2007). This was also the case for the forests in our dataset 414 (Table 1 and 2). Higher wood relative to foliage production may thus increase the Ra-to-GPP 415 ratio in forests of high nutrient availability compared to forests of low nutrient availability. 416 Further, several studies show a positive relation between root respiration per unit mass and root 417 nutrient concentrations (Chapin 1980; Burton et al. 2002), but this may be counterbalanced by a 418 decrease in standing root biomass as indicated by the negative fertilization effect on root 419 respiration found in a recent meta-analytical study (Janssens et al. 2010).

421 While the effects of nutrient availability on the ratio of Ra to GPP remain unclear, a decrease in 422 the Ra-to-GPP ratio for forests with high nutrient availability relative to forests of low nutrient 423 availability seems unlikely according to the theory that plants respire a relatively constant 424 fraction of GPP (Dewar et al. 1998; Van Oijen et al. 2010) due to interdependencies of 425 respiration and photosynthesis (Hoefnagel et al. 1998).

426. 


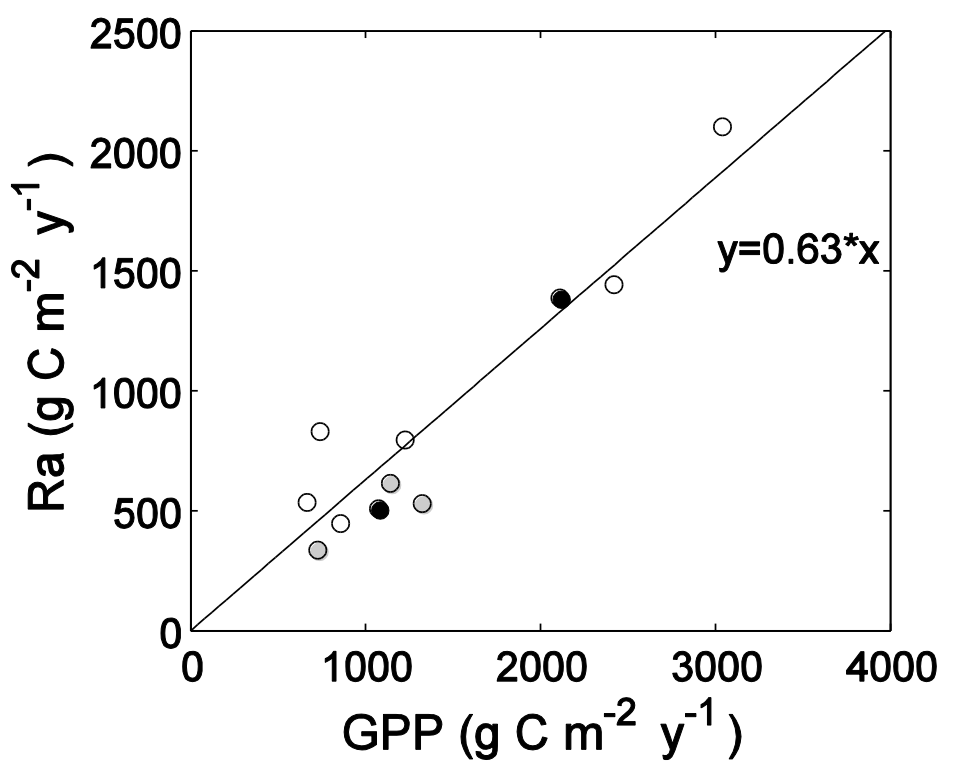

428 Figure 3: Field estimates of autotrophic respiration ( $\mathrm{Ra}$ ) versus gross primary production (GPP).

429 Each single data point represents one forest site and is the average value over all years for which 430 data were available in the database. White, gray and black circles indicate sites of low-, medium431 and high-nutrient availability, respectively. The equation refers to the linear fit through the data.

432 One nutrient-poor site with Ra:GPP>1 was removed. We found no statistically significant 433 nutrient-availability effect on Ra:GPP ( $p=0.34$ ), but these results remain tentative due to the 434 small number of data points 
437 In this study, we identified a gap in the current knowledge of forest carbon allocation: forests 438 with high nutrient availability use $16 \pm 4 \%$ more of their photosynthates for biomass production 439 than forests with low nutrient availability (16 $\pm 4 \%$ represents the difference between mean of 17 440 forests of high nutrient availability and mean of 19 forests of low nutrient availability; SE 441 calculated as $\mathrm{SE}_{\text {difference }}=\operatorname{sqrt}\left(\mathrm{SE}_{1}{ }^{2}+\mathrm{SE}_{2}{ }^{2}\right)$, with $\mathrm{SE}_{1}$ and $\mathrm{SE}_{2}$ the $\mathrm{SE}$ for low- and high nutrient 442 availability, respectively). This difference is, however, unlikely attributable to a difference in 443 carbon partitioning to autotrophic respiration. It therefore appears likely that forests of low 444 nutrient availability invest more photosynthates in non-biomass components of net primary 445 production (NPP), which usually are not quantified in experiments or reported in the literature 446 and therefore could not be taken into account in our analysis. These unaccounted for NPP 447 components include volatile organic compounds, root exudates, and root symbionts. We 448 hypothesize that carbon allocation to root symbionts in particular is a key factor explaining the 449 higher biomass production efficiency in nutrient-rich relative to nutrient-poor forests. First 450 support for this hypothesis is given in literature:

452 Symbiotic fungi are essential for the growth and health of forest trees (Courty et al. 2010), as 453 they transport nutrients from soil to tree (van der Heijden et al. 2008; Courty et al. 2010). Up to $45480 \%$ of plant nitrogen and $75 \%$ of plant phosphorus can be fungal-derived in forests (van der 455 Heijden et al. 2008). In return for these nutrients, considerable amounts of carbon are transferred 456 from tree to fungus (van der Heijden et al. 2008; Courty et al. 2010). Recent reviews (Hobbie 457 2006; Courty et al. 2010), mostly based on controlled short-term studies, state that the overall 458 carbon flux to mycorrhizal fungi can constitute up to $30 \%$ of NPP (but observational estimates 
459 remain scarce and highly variable; see Hobbie 2006 and Courty et al. 2010). Nonetheless, one 460 long-term field study in a nutrient-rich, temperate oak forest (Heinemeyer et al. 2012) where the 461 mycorrhizal soil carbon flux contribution was estimated at about $20 \%$ of NPP, confirms this 462 order of magnitude.

464 It has been shown repeatedly that carbon transfer to fungal symbionts are strongly inversely 465 related to nutrient availability (Wallenda \& Kottke 1998; Lilleskov et al. 2002; Högberg et al. 466 2003; Read \& Perez-Moreno 2003; Treseder 2004; Högberg et al. 2010), opening the door for a 467 substantial effect on the biomass production efficiency (BPE). According to a meta-analytical 468 review, mycorrhizal abundance declines substantially in response to nitrogen and phosphorus 469 fertilization (15\% and 32\%, respectively) (Treseder 2004). Similar responses were observed 470 along natural gradients in nutrient availability. Both biodiversity and proteolitic capabilities of

471 ectomycorrhizal fungi declined along a gradient of increasing mineral nitrogen availability 472 through Europe (Schulze 2000), phospholipid fatty acid attributed to mycorrhizal fungi 473 dramatically decreased along a natural soil nitrogen gradient in a boreal forest (Högberg et al. 474 2003), and both taxonomic richness and sporocarp abundance decreased over an anthropogenic 475 nitrogen deposition gradient in Alaska (Lilleskov et al. 2002).

477 Following this well-reported and strong relation between root symbionts and nutrient availability, 478 we hypothesize that forests with high nutrient availability produce more biomass per unit 479 photosynthesis than forests with low nutrient availability because the latter need to invest 480 relatively more photosynthates in root symbionts. 


\section{Tables}

483 Table 1: Mean and standard deviation (in brackets) for gross primary production (GPP), total 484 biomass production (BP) and the biomass production-to-GPP ratio (BPE) in boreal, temperate 485 and tropical forests of different nutrient availability (low, medium and high). For statistics, see 486 Table 3. The number of forests per group is indicated in Figure 2A.

487

488

489

490

491 Temperate, low

GPP

BP

BPE

492 Tropical, low

$1320(718)$

493 Boreal, medium

2985 (591)

1233 (315)

$0.41(0.11)$

494 Temperate, medium

803 (204)

390 (112)

$0.49(0.10)$

495 Temperate, high

1328 (372) $\quad 659(208)$

$0.50(0.11)$

$1724(408) \quad 1008(354)$

$0.58(0.13)$

496

497 
498 Table 2: Mean and standard deviation (in brackets) for the ratio of belowground to aboveground 499 biomass production (BBP:ABP), aboveground wood production (AWP), foliage production (FP), 500 root production (RP) and the ratio of aboveground wood production to gross primary production 501 (AWP:GPP) in boreal, temperate and tropical forests of different nutrient availability (low, 502 medium and high). For statistics, see Table 3. The number of forests per group are indicated in 503 superscript.

504

505 Climate zone,

BBP:ABP

AWP

FP

$\mathbf{R P}$

AWP:GPP

506 nutrient availability

$\left(\mathrm{g} \mathrm{C} \mathrm{m}^{-2} \mathbf{y}^{-1}\right)$

$\left(\mathrm{g} \mathrm{C} \mathrm{m}^{-2} \mathbf{y}^{-1}\right)$

(g C m m $\left.^{-2} \mathbf{y}^{-1}\right)$

507

Boreal, low

$0.65(0.29)^{5} \quad 100(46)^{5}$

$61(24)^{5}$

$125(65)^{5}$

$0.11(0.02)^{5}$

508 Temperate, low

$0.66(0.31)^{6} \quad 166(80)^{6}$

$153(98)^{6}$

$205(97)^{6}$

$0.13(0.03)^{6}$

509 Tropical, low

$0.28(0.09)^{5} \quad 348(85)^{5}$

$404(151)^{5}$

$282(47)^{5}$

$0.11(0.03)^{5}$

510

Boreal, medium

$0.45(0.27)^{5}$

$116(32)^{5}$

$72(36)^{5}$

$117(68)^{5}$

$0.15(0.03)^{5}$

511 Temperate, medium

$0.88(0.90)^{7} \quad 212(129)^{6}$

$149(97)^{7}$

$238(122)^{7}$

$0.14(0.07)^{6}$

512 Temperate, high

$0.33(0.17)^{17}$

$493(335)^{16}$

$184(50)^{16}$

$218(88)^{17}$

$0.27(0.14)^{16}$

513

514 
515 Table 3: Statistical analysis for gross primary production (GPP), biomass production (BP), the 516 biomass production-to-GPP ratio (BPE), the ratio of belowground to aboveground biomass 517 production (BBP:ABP), aboveground wood production (AWP), foliage production (FP), root 518 production (RP), and the wood production-to-GPP ratio (AWP:GPP). The column 'stepwise fit' 519 indicates the predictor variable(s) (climate zone $(\mathrm{C})$, forest type $(\mathrm{F})$, management $(\mathrm{M})$, stand age 520 (A), nutrient availability $(\mathrm{N})$ ) selected by the stepwise regression at $\mathrm{p}<0.05$. ANOVA(1) shows 521 results of ANOVA with the variables selected by the stepwise regression as fixed factors (or as 522 covariable in case of stand age). ANOVA(2) gives results of a two-way ANOVA with climate 523 zone and nutrient availability as fixed variables and thus corresponds to data shown in Tables 1 524 and 2.

525

526

Variable

Stepwise fit

C, $\mathrm{N}$

$527 \quad$ GPP

528

529

530

531

532

533

534

535

536

$537 \quad$ FP

538

539

540
BP

A, C, N

BPE

$\mathrm{N}, \mathrm{M}$

BBP:ABP N

AWP

C, N

$\mathrm{C}, \mathrm{F}$

C

$\mathrm{N}$

\section{$\operatorname{ANOVA(1)}$}

Boreal $<$ Temperate $<$ Tropical $(\mathrm{p}<0.01)$

Nutrients: low=medium $<$ high $(\mathrm{p}=0.05)$

Boreal $<$ Temperate $<$ Tropical $(\mathrm{p}<0.01)$

Nutrients: low=medium $<$ high $(\mathrm{p}<0.01)$

negative age effect $(\mathrm{p}=0.01)$

low=medium $<$ high $(\mathrm{p}<0.01)$

Unmanaged $<$ Managed $(\mathrm{p}=0.07)$

Nutrients: low=medium $>$ high $(\mathrm{p}=0.07)$

Boreal=Temperate $<$ Tropical $(\mathrm{p}<0.01)$

Nutrients: low=medium $<$ high $(\mathrm{p}<0.01)$

Boreal $<$ Temperate $<$ Tropical $(\mathrm{p}<0.01)$

Needle-leaved $<$ broadleaved $(\mathrm{p}<0.01)$

Boreal $<$ Temperate $=$ Tropical $(\mathrm{p}<0.01)$

Nutrients: low=medium $<$ high $(\mathrm{p}<0.01)$
$\operatorname{ANOVA}(2)$

$\mathrm{C}: \mathrm{p}<0.01 ; \mathrm{N}: \mathrm{p}=0.05$

$\mathrm{C}: \mathrm{p}<0.01 ; \mathrm{N}: \mathrm{p}<0.01$

$\mathrm{C}: \mathrm{p}=0.69 ; \mathrm{N}: \mathrm{p}<0.01$

$\mathrm{C}: \mathrm{p}=0.69 ; \mathrm{N}: \mathrm{p}<0.01$

$\mathrm{C}: \mathrm{p}<0.01 ; \mathrm{N}: \mathrm{p}<0.01$

$\mathrm{C}: \mathrm{p}<0.01 ; \mathrm{N}: \mathrm{p}=0.13$

$\mathrm{C}: \mathrm{p}<0.01 ; \mathrm{N}: \mathrm{p}=0.92$

$\mathrm{C}: \mathrm{p}=0.94 ; \mathrm{N}: \mathrm{p}=0.01$ 


\section{$541 \quad$ Figures}

542 Figure 1: Each circle represents the mean annual total biomass production \pm SE versus mean

543 annual gross primary production $(\mathrm{GPP} \pm \mathrm{SE})$ for one forest. Colours indicate nutrient availability

544 classes, error bars reflect uncertainties (see Appendix S1). Dotted, dashed and solid lines are

545 linear fits $(\mathrm{y}=\mathrm{ax})$ for the low-, medium- and high nutrient availability class, respectively

$546 \quad\left(\mathrm{R}^{2}=0.84, \mathrm{R}^{2}=0.66, \mathrm{R}^{2}=0.56\right.$, respectively; $\mathrm{p}<0.01$ for low- versus high nutrient availability (GLM

547 analysis)). The squares on the right represent the mean biomass production efficiency (BPE:

548 biomass production-to-GPP ratio). Error bars on these squares are standard errors on the means,

549 reflecting measurement uncertainties and inter-annual variability in case of multi-year data.

550 Letters next to the squares indicate significant differences at $\mathrm{p}<0.05$ (Tukey post-hoc test;

551 ANOVA with nutrient availability as fixed factor).

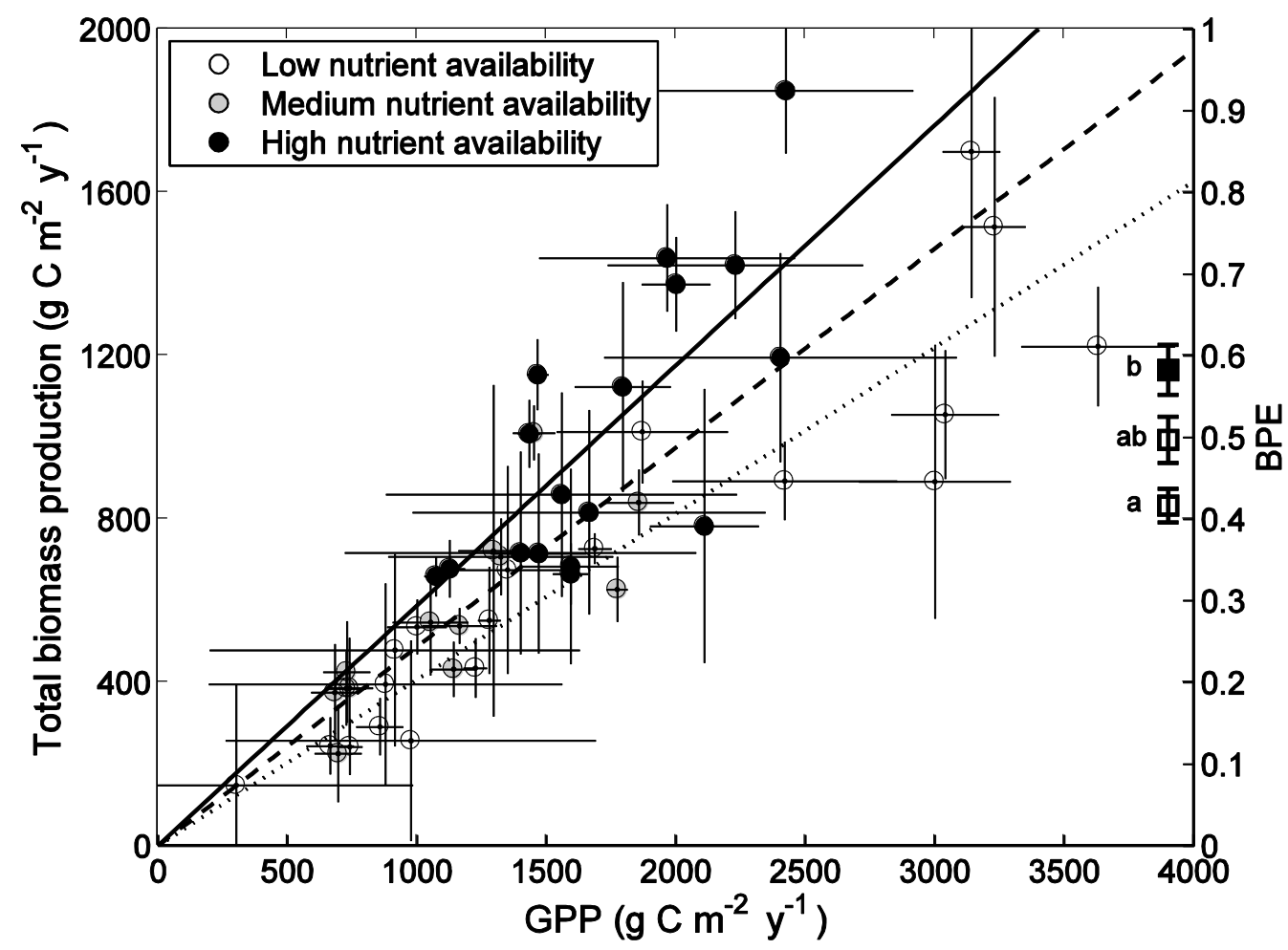


554 Figure 2: Mean biomass production efficiency (BPE) versus nutrient availability class for (A)

555 different climate zones, (B) management practices, (C) forest types and (D) BPE versus stand age

556 for the three nutrient availability classes. Error bars represent the standard error on the mean and

557 numbers indicate the number of forests per group. Stepwise regression analysis revealed a

558 significant effect of nutrient availability $(\mathrm{p}<0.01)$ and forest management $(\mathrm{p}=0.02)$. Climate zone,

559 forest type and stand age were not statistically significant $(\mathrm{p}>0.1)$. Note that for six forests no

560 estimate for stand age was available and these sites were thus omitted from this analysis.

561 Removing stand age from the regression model, which allows inclusion of these six sites, did not

562 alter the outcome (data not shown).

563
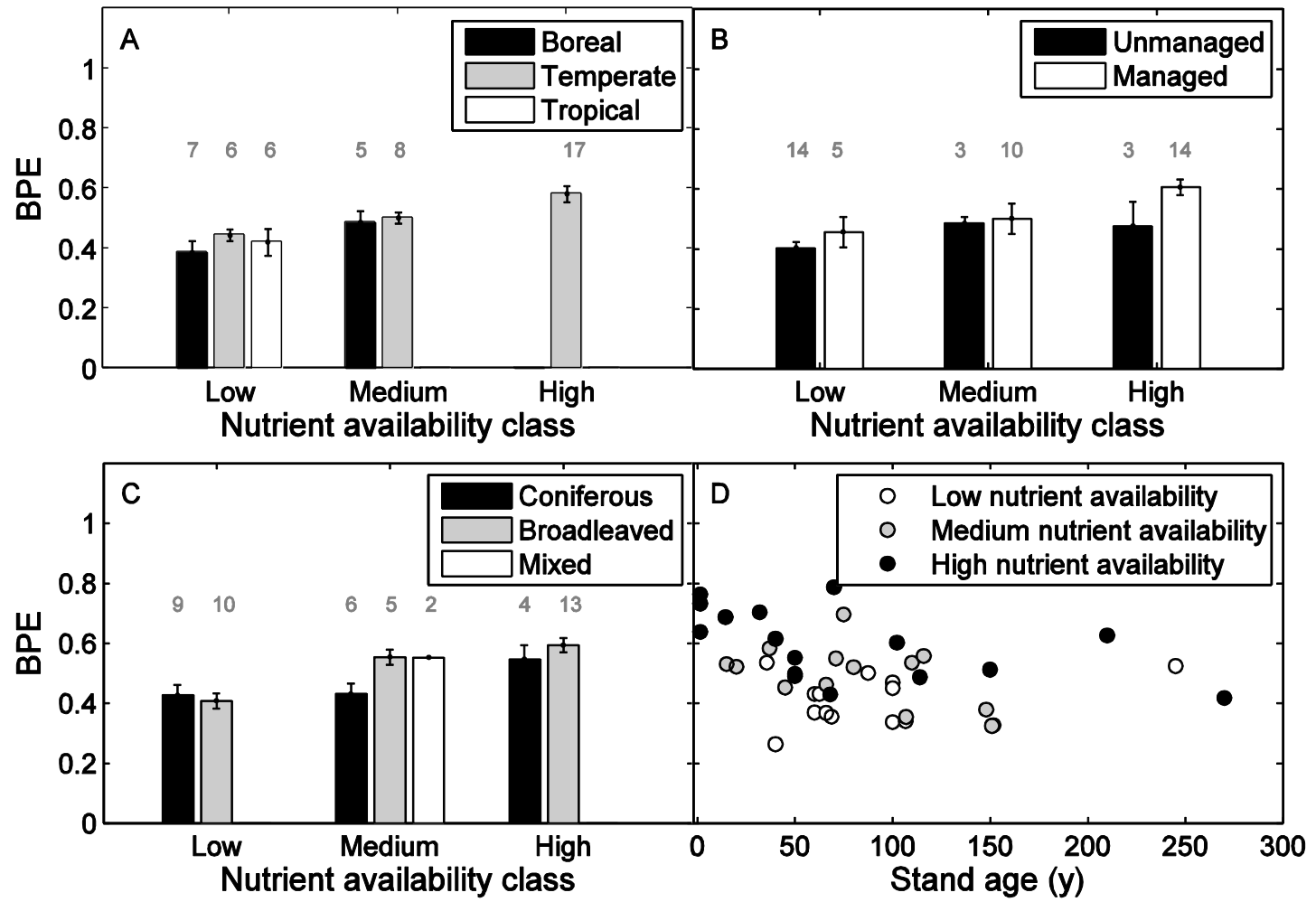

564 